\section{Differential luminance sensitivity of the human visual system*}

\author{
J. M. THIJSSEN $\dagger$ and A. J. H. VENDRIK $\dagger \dagger$ \\ Nijmegen University, Nijmegen, The Netherlands
}

In this paper the differential sensitivity of the visual system is investigated by means of two simultaneously presented stimuli in a yes-no procedure. The sensitivity measure $\sigma_{I}$ appears to be proportional to stimulus intensity (i.e., Weber's law). The curve displaying Weber's law is little affected by variation of the background intensity or of the adaptation level. An increment threshold experiment using only one stimulus yields a proportionality of $\sigma_{\mathrm{I}}$ with the square root of the background intensity. An additional experiment shows that the sensitivity measure $\sigma_{I}$ for two flashes decreases first, from dark up to a particular background intensity, and increases when the background tends to mask the flashes. So, in general, two background levels exist with the same differential sensitivity. The results cannot easily be explained by the simple quantum fluctuation concept. A model based partially on electrophysiological data from the literature is proposed which encounters a particular adaptation mechanism, a transducer with a limited dynamical range, and a range setting mechanism.

Several theoretical models have been proposed by a number of authors in order to describe the differential sensitivity of human senses. Various forms of threshold models have been developed, all of them assuming that sensitivity is limited by a fixed threshold. A thorough discussion of these models was given by Green and Swets (1966). In the past decade the theory of signal detection has been successfully applied to sensory detection by human Os. This theory assumes that differential sensitivity is limited by noise and that the 0 adopts a detection criterion. This criterion can be shifted by the $O$ to reach optimum performance in a particular experimental set-up. Many experiments were carried out to investigate the applicability of the theory by means of changing the signal-to-noise ratio of the stimulus (Swets, 1964). Although this kind of experiment yielded interesting information about the central nervous decision mechanism, it may be not the most straightforward method to investigate the properties of a sensory system.

Another procedure was performed by Eijkman and Vendrik (1963). By using stimuli with a noise level as low as possible they showed that the theory of signal detection is useful to describe the experimental data of the measurement of the absolute sensitivity of touch and

*This paper is based in part on a dissertation for the PhD in the Faculty of Science, Nijmegen University, Nijmegen, The Netherlands.

†Present address: Laboratory of Physics, Department of Ophthalmology, Nijmegen University, Geert Grooteplein Z 22, Nijmegen, The Netherlands.

$\dagger \dagger$ Department of Medical Physics, Nijmegen University, Geert Grooteplein N 21, Nijmegen, The Netherlands. study it was assumed that absolute sensitivity is limited by internal noise, which was proposed to be a fluctuating neural activity added to the nonfluctuating neural activity evoked by the stimulus. Eijkman and Vendrik showed that certain characteristics of this internal noise can be derived.

Eijkman et al (1966) studied the differential sensitivity of the auditory system by using stimuli without purposely added noise. Differential sensitivity is defined as the intensity increment which yields a particular degree of discriminability from the original stimulus. They investigated the validity of Weber's law, which states that differential sensitivity is proportional to stimulus intensity. Eijkman et al (1966) studied the hypothesis that Weber's law can be explained by multiplicative noise. This noise is assumed to originate from fluctuations of a gain factor in the sensory s;'stem and thereby increases with stimulus strength. A detection model for the auditory system based upon this hypothesis has been called the "multiplicative noise model." It appeared, however, that the experimental results could be described better by another model, which has been called the "multirange meter model." In this model the relevant internal noise is of an additive nature. To explain Weber's law it has been assumed that a gain factor within the sensory system is adapted to the range of intensities used. This gain-adapting mechanism may be considered to be a kind of habituation effect. The $O$ focuses his attention on the range of stimulus intensities in order to be able to use a subtle discrimination system with a limited warmth perception of the skin. In that dynamical range, in contrast to a coarse system, which may be used in case of unknown stimuli. The consequence of this range-setting mechanism is that, if a constant noise power is present beyond it, the noise "translated" to the input becomes signal dependent, i.e., dependent on the overall level of stimulus intensities used in a particular experiment, but not on the actual value of the stimulus intensity in a single trial.

Thijssen and Vendrik (1968) evaluated the consequences, which result from the assumptions included in both of the above-mentioned detection models, on psychometric curves and ROCs (receiver operating characteristics). These two types of curves represent two possible ways of elaborating the data of discrimination experiments. They showed how the applicability of the models can be investigated by studying the properties of the curves. Moreover, they confirmed the result of Eijkman et al (1966) regarding the preference of the multirange meter model for the auditory system. The transducer function relating stimulus intensity with neural activity, upon which a decision is based, was shown to be intensity dependent. This result has been confirmed independently by Treisman and Irwin (1967), who compared monaural and binaural detection. Thijssen and Vendrik (1968) also presented data on visual discrimination. The conclusion was that for the visual system the multirange meter model is to be preferred, i.e., the noise limiting the discrimination sensitivity is additive.

The present paper concerns an investigation of the applicability of Weber's law to the differential sensitivity of the visual system under various conditions of the adaptation level. The conventional method of increment threshold determination is not adequate from detection theoretical point of view. With this method it is assumed that the $\mathrm{O}$ is able to take a sample identical both in duration and location with the stimulus from a continuously presented background, which has not been proven to be true (cf. Leshowitz et al, 1968). Moreover, in the interpretation of the data it is often assumed that the transducer function is equal for background light and flash light (Treisman, 1966; Treisman \& Leshowitz, 1969). The latter assumption is unlikely, as can be concluded both from psychophysical and electrophysiological evidence (Craik, 1940; Stevens \& Stevens, 1963; Sakman \& Creutzfeld, 1969). The present experiments are designed to study the effect of stimulus intensity and background level or adaptation level separately. The stimuli were accompanied 


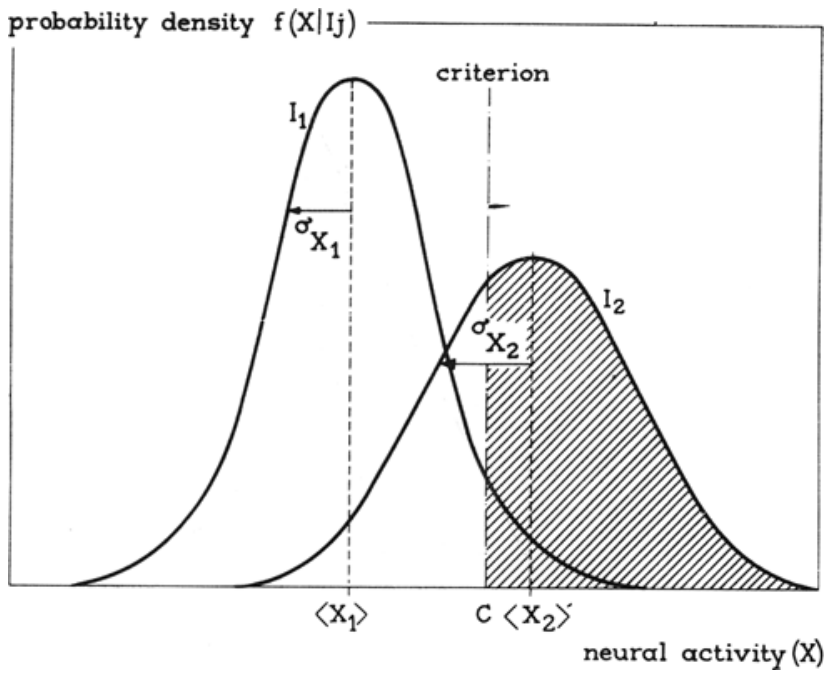

Fig. 1. Probability density curves for multiplicative Gaussian noise $\left[\sigma_{X}(:) X\right]$. The shaded area corresponds with $\mathbf{P}\left(\mathbf{R}_{2} \mid \mathbf{I}_{2}\right)=\mathbf{P}\left(\mathrm{X}_{2}>\mathbf{C}\right)$.

with a reference stimulus of identical area and duration, but at a different location. This procedure results in a differential sensitivity measurement with two equivalent stimuli. Comparison with conventional increment threshold measurements is made possible by means of an additional series of this kind of experiment.

The multirange meter model will be extended in order to include the effect of variation of the adaptation level. It may be noted beforehand that the range-setting mechanism proposed in the model is very similar to the explanation of light adaptation given by Craik (1940). Therefore, it can be expected that the multirange meter model will be made adequate with a little modification. With a particular assumption about the transducer function it appears that the model predicts Weber's law for the relation between the differential sensitivity and the stimulus intensity (see Appendix).

\section{PSYCHOPHYSICAL METHODS}

The stimuli presented to the $O$ are a test stimulus and a simultaneously given reference stimulus. In each experiment, at least four values of test stimulus intensity are used. These test stimulus categories are presented in a random sequence.

In a detection experiment the $\mathrm{O}$ is asked to state whether or not the test stimulus is more intense than the reference stimulus. The procedure will be illustrated now in terms of the theory of signal detection for the most simple case of two test stimuli. The neural activity evoked by a stimulus which is relevant to a decision will be denoted by $X$. This neural activity may be the result of Stimulus 1 with Intensity $I_{1}$ or of Stimulus 2 with Intensity $I_{2}$ $\left(\mathrm{I}_{2}>\mathrm{I}_{1}\right)$. $\mathrm{X}$ is a fluctuating quantity. As the $O$ has to state whether $I_{1}$ or $I_{2}$ has been presented, the strategy is as follows: If the observed magnitude of $\mathrm{X}$ exceeds a particular level, $\mathrm{C}, \mathrm{O}$ should always respond that Stimulus 2 is observed (Response $R_{2}$ ). With a large number of trials, the relative frequency of a response is approximately equal to the probability of occurrence. So $P\left(R_{2} \mid I_{j}\right)$ and $P\left(R_{1} \mid I_{j}\right)$, with $\mathrm{j}=1$ or 2 , will be obtained. It is clear that $P\left(R_{1} \mid I_{1}\right)+P\left(R_{2} \mid I_{1}\right)=1$; the same relation holds for $l_{2}$. Figure 1 shows the probability density curves of $X$ ( $f=$ probability density) corresponding to
Stimuli 1 and 2, respectively. In this figure it is assumed that the noise is multiplicative and Gaussian distributed. The shaded area in Fig. 1 corresponds with $P\left(R_{2} \mid I_{2}\right)$, so $P\left(R_{2} \mid I_{2}\right)$ equals

$$
P\left(X_{2}>C\right)=\int_{C}^{+\infty} f\left(X \mid I_{2}\right) d X
$$

In a detection experiment with five different test stimuli, the response probabilities $P\left(R_{2} \mid I_{j}\right)$, with $j=1$ to 5 , are used to measure the differential sensitivity. For that purpose the probabilities are plotted vs stimulus intensity. The resulting graph is called a psychometric curve. A quantity $\sigma_{\mathrm{I}}$ is defined, which is a convenient measure of the differential sensitivity (Fig. 2). $\sigma_{1}$ equals the stimulus intensity range that corresponds to the set of probabilities $(0.84 ; 0.50)$. This probability region encloses the standard deviation, $\sigma_{X}$, of the neural activity, $X$, only in case of additive Gaussian noise and a linear transducer (Thijssen \& Vendrik, 1968).

To investigate the validity of Weber's law and the influence of light adaptation on the sensitivity of the parafoveal region, four series of experiments were performed. The various series can be listed as follows: Series $A$. Discrimination between a reference flash and a test flash, presented simultaneously, the eye being dark-adapted. The trials are separated by a dark interval of at least $10 \mathrm{sec}$. Series $B$. Detection of a test stimulus delivered on a continuously presented background. Five different background intensities are used.

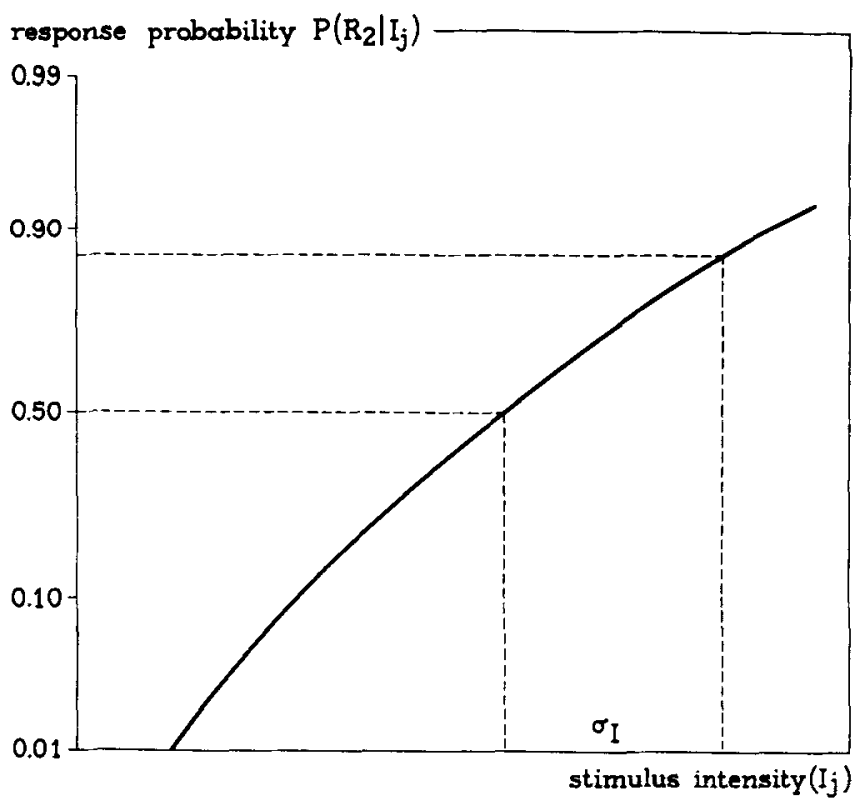

Fig. 2. The value of $\sigma_{I}$ is defined also for a curved psychometric curve as the intensity region that corresponds with the response probability interval, 0.50 to 0.84 . 


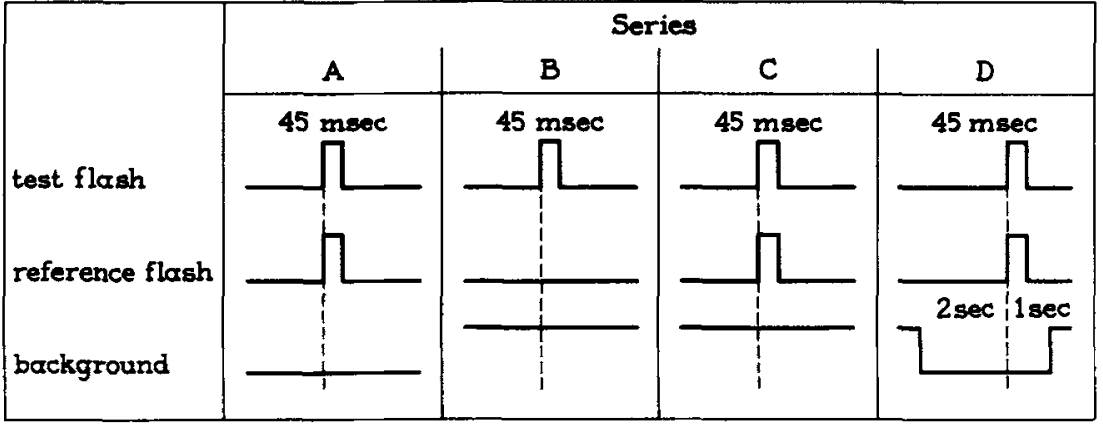

Fig. 3. Stimulus and background conditions for Series A, B, C, and D. The switching time of the background of Series $D$ is not to scale with the stimulus duration. In Series $A$ the eye remains dark adapted.

Series C. Discrimination between a and D will permit a conclusion as to reference flash and a test flash delivered on whether or not the presence of the the same background levels as in B. background is an important factor in Series $D$. Discrimination between a adaptational changes of the sensitivity.

reference flash and a test flash; the background is switched off $2 \mathrm{sec}$ before until $1 \mathrm{sec}$ after the display of the flashes, and remains on for $10 \mathrm{sec}$. A repetition of one trial per $13 \mathrm{sec}$ results. The same background levels as in $B$ are used.

The time sequence of the experiments is shown in Fig. 3. The Os were dark-adapted for at least $30 \mathrm{~min}$ and, if necessary, light-adapted for $5 \mathrm{~min}$.

In Series A, C, and D five test stimuli are delivered at random. Two of these stimuli are less intense, two are more intense, and one equals the reference stimulus. In Series B three test stimuli of different intensity are mixed at random with stimuli of zero intensity in a proportion of $60 \%$ to $40 \%$, respectively. The Os have to state whether or not a test flash is presented (Fig. 3). The reference flash in Series B is always clearly visible, and thus an $O$ will be less uncertain about the localization of the test flash. The stimulus intensities of Series $C$ are such that the level of the reference flash is just $100 \%$ visible. The intensity of the flashes of Series A is chosen such that the reference flashes equal those of Series $D$.

Series B represents the orthodox way of measuring the "increment threshold." Thus, it is possible to compare the results of other Es with the present series. However, there is a difference in the measure of sensitivity used in this study and the increment threshold. The increment threshold may be defined as the increment $\Delta \mathrm{I}$ corresponding to a probability of seeing, e.g., 0.50 . In this study, $\sigma_{I}$ is used (see Fig. 2). This difference will be discussed further on.

Comparison of Series A and D provides the opportunity to investigate the influence of a change of state of adaptation of the retina on the sensitivity, whereas comparison of the results of Series A, C, position.

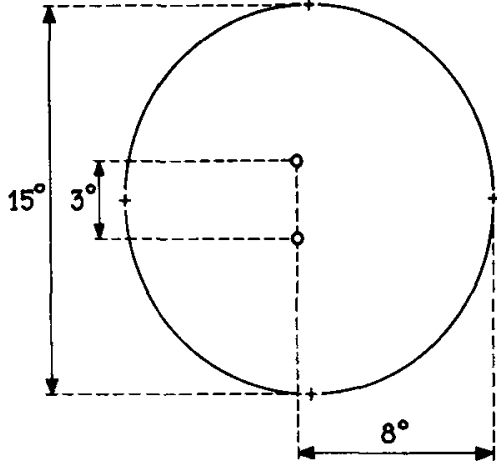

Fig. 4. The field observed by the 0 with the right eye. The $O$ is instructed to fixate the right-hand cross. The three other crosses should be visible by foveal inspection at the proper head position. The flashes subtend $10 \mathrm{~min}$ of arc and are separated by 3 deg.

\section{EXPERIMENTAL ARRANGEMENT}

The experiments were carried out with the following setup. Two flashes separated by $3 \mathrm{deg}$ of visual angle are presented simultaneously. The flash area is $10 \mathrm{~min}$ and the duration is $45 \mathrm{msec}$. The flashes are accompanied by a $45-\mathrm{msec}, 1,000-\mathrm{Hz}$ tone pip, in order to support the attentiveness of the 0 . According to Bouman and van den Brink (1952) and Denton and Pirenne (1952), a separation of $3 \mathrm{deg}$ will suffice to avoid summation effects of the flashes in the parafoveal region. The flashes are presented at $8 \mathrm{deg}$ in the nasal field of the right eye, i.e., at $8 \mathrm{deg}$ temporal retina. According to Zigler

and Wolff (1958), little variation of sensitivity occurs in this region of the retina. The background is a circular field with a diameter of $15 \mathrm{deg}$. Fixation is ensured by means of four small red crosses at the border of the background field. The four crosses are positioned like a large cross, and the $O$ has to fixate the right-hand cross. With this configuration the head is in the exact position only if all the crosses can be seen by foveal inspection. Additionally, the head is positioned by means of a chinrest and foreheadrest. The view of the $O$ is shown in Fig. 4. Both a 3-mm and a 4-mm artificial pupil are used in the experiments.

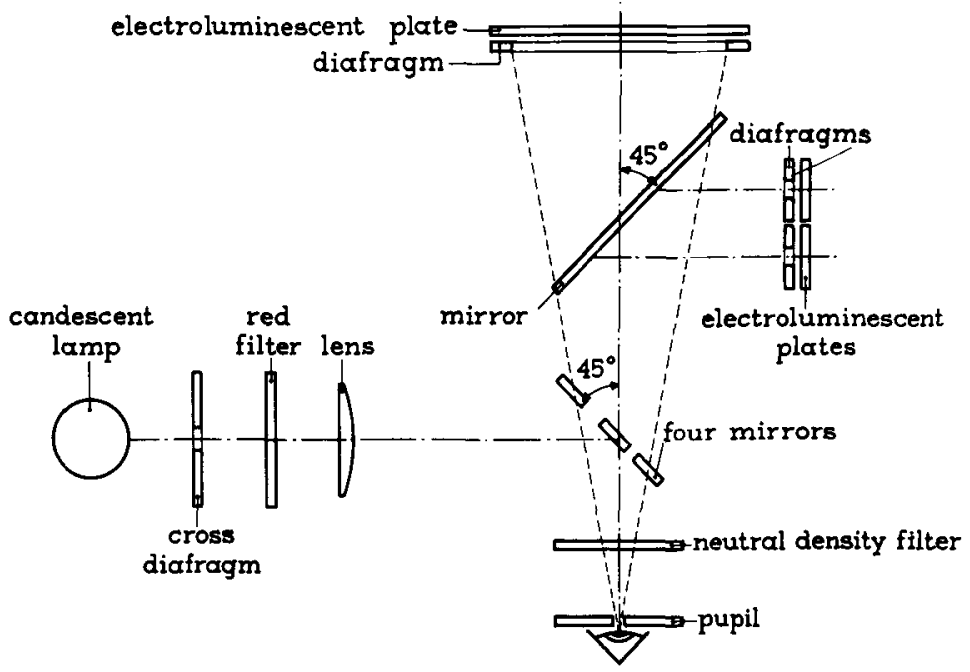

Fig. 5. The experimental arrangement. The 0 looks at the light sources through a 3- or 4-mm artificial pupil. The luminescent plates are placed at $50 \mathrm{~cm}$ from this pupil; hence, the $\mathrm{O}$ has to accommodate 2 diopters. The positive lens depicts a virtual image of a cross diaphragm at a distance of $50 \mathrm{~cm}$ from the artificial pupil. The light is reflected by four small mirrors, which yield the fixation configuration in Fig. 4. The whole system is placed in a light-tight box. The 0 looks into the box through a tube (length, $15 \mathrm{~cm}$ ). This tube limits the visual field, and the four fixation crosses can be seen only at the exact head 


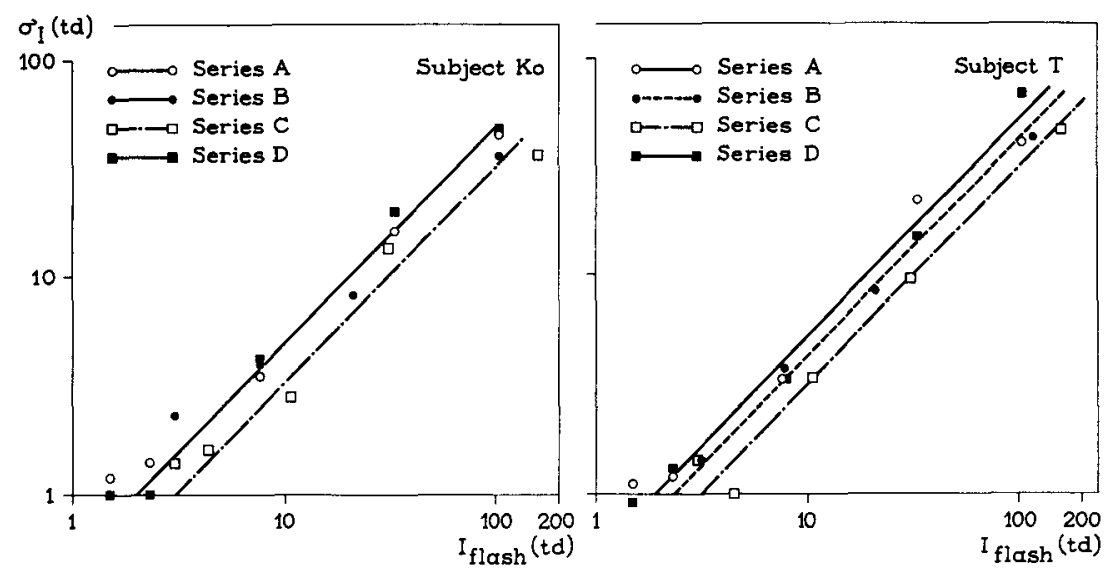

Fig. 6. $\sigma_{\mathrm{I}}$ plotted vs the intensity of the central flash level. For Series B the lowest flash level has been plotted on the abscissa. All series display a proportionality of $\sigma_{I}$ with Iflash, in first approximation.

Background field and flashes are made by means of electroluminescent plates (Philips, EL $20011 / 009$ ), driven by a $1,000-\mathrm{Hz}$ voltage. The apparatus is shown in Fig. 5. The stimuli are produced and the responses are rated automatically (Thijssen, 1969).

The background levels used in Series B, $C$, and $D$ comprise an intensity range of 0.08 to $180 \mathrm{td}$. This range is localized mainly in the mesopic region, which, according to Pirenne (1962), comprises the range $10^{-3}$ to $10 \mathrm{~cd} / \mathrm{m}^{2}$, or $4 \times 10^{-2}$ to $10^{2} \mathrm{td}$. Each experiment of Series B and C consists of 200 trials; in Series A and D 100 trials per experiment were carried out.

\section{RESULTS}

For all the series the probability of the statement "the test flash exceeds the reference flash" or "exceeds the background level" [i.e., $\left.P\left(R_{2} \mid I_{j}\right)\right]$ is plotted on a normal probability scale vs test flash intensity on a linear scale. This procedure yields one psychometric curve. Most of the curves have been approximated with a single straight line. The value of $\sigma_{\mathrm{I}}$, i.e., the intensity range corresponding to the standard deviation, is measured. For clearly curved lines the slope is determined by averaging the slope of the straight lines through the lower and the higher points. The experiments of Series A, B, and D were performed on the same days. A second Series B was carried out together with Series $C$.

In Fig. $6 \log \sigma_{I}$ is plotted against log flash intensity. For Series $A, C$, and D, the intensity of the reference flash has been taken, whereas for Series B the flash intensity corresponding to a response probability $P\left(R_{2} \mid I_{j}\right)=0.50$ has been used. The data can be approximated by a straight line with unity slope. It can be seen in Fig. 6 that all the series display a proportionality resulting in Weber's law. The proportionality constant does not depend much on the presence of a background, i.e., Series $A$ and $D$ as compared with Series B and C. The influence of the adaptation level, which was zero for Series $A$ and varying for Series D, seems not to be large either. The conclusion is that differential sensitivity for the discrimination of two flashes is determined primarily by the intensity of the flashes.

The influence of the background level on the discriminability of two flashes was investigated further by means of experiments of Type $\mathrm{C}$, but now with constant flash level and with variation of the background intensity over the range of 0 to $350 \mathrm{td}$. The results for two Os are shown in Fig. 7. The reference flash had an intensity of $175 \mathrm{td}$. As can be seen, the best performance is obtained at an intermediate background level, whereas in the dark (cf. Series A) and at high level (cf. Series B) discrimination is worse.

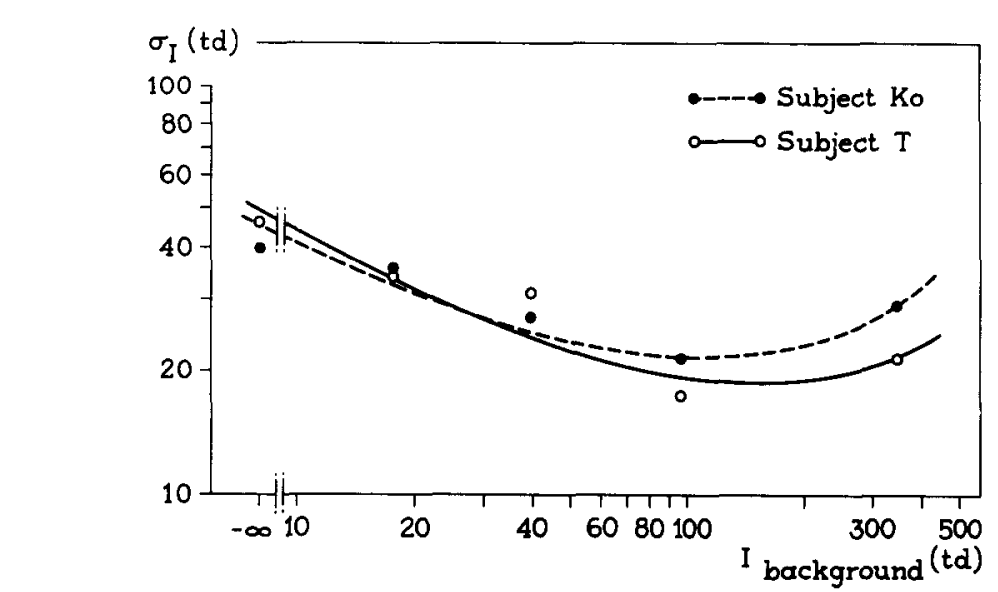

Fig. 7. With fixed stimulus intensities, $\sigma_{1}$ appears to decrease first and to increase later
increasing the background intensity from 0 to about $400 \mathrm{td}$. The intensity of the

Fig. 7. With fixed stimulus intensities, $\sigma_{1}$ appears to decrease first and to increase later
by increasing the background intensity from 0 to about 400 td. The intensity of the reference stimulus equals $175 \mathrm{td}$. the higher parts of the curve (the dots in Fig. 8), and secondly, by taking the slope of the higher part only (the triangles in Fig. 8). The data of Series $C$, which also display a square-root relation, can be understood by the result shown in Fig. 6. The flash intensities of Series $C$ are a constant factor larger than those of Series B. Hence, the $\sigma_{I}$ values of Series C will differ also by a constant factor from those of Series B, and therefore Series C will display the same relationship between $\sigma_{I}$ and background intensity as does Series B.

In conclusion, the proportionality of $\sigma_{I}$ to flash intensity for the discrimination of two flashes is affected neither by variation of the adaptation level of the retina nor by the actual presence of a background intensity. The proportionality constant (Weber constant) varies slightly with varying the background intensity level from zero to a level at which the flashes tend to become masked by the
The course of the curves of Fig. 7 corresponds to the shift of the $\sigma_{I}$ values at each $I_{f l a s h}$ of Series $C$ with respect to those of Series A and B, as displayed in Fig. 6.

The results of Series $B$ can be worked out in a plot of $\sigma_{I}$ vs background intensity in order to check the commonly observed square-root relation between increment threshold and background intensity for detection of small short flashes on a continuously presented background (cf. Bouman, 1952; and Barlow, 1957). The data are shown in Fig. 8, and it can be seen that a square-root relation fits fairly well. The slope of the psychometric curves of Series B increases with increasing flash intensity. Therefore, the value of $\sigma_{\mathrm{I}}$ is determined in two ways: first, by taking the average of the slope of the lower and Perception \& Psychophysics, 1971, Vol. 10 (1) 


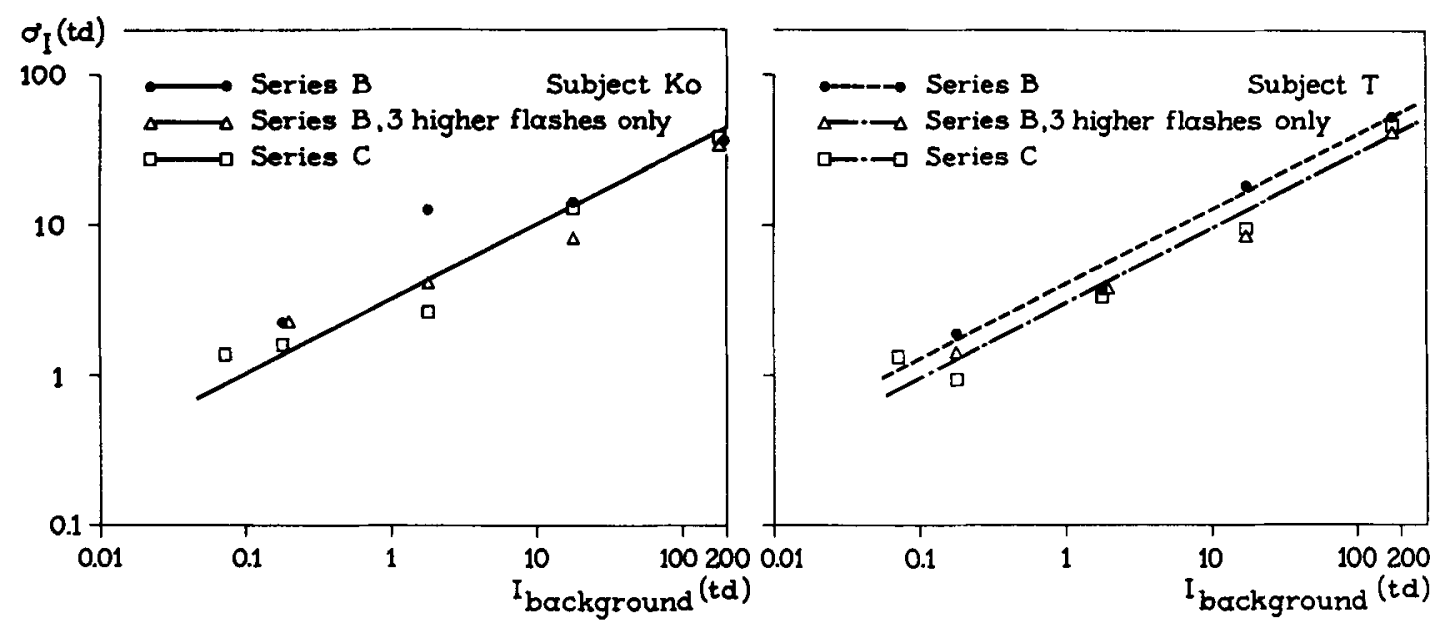

Fig. 8. $\sigma_{1}$ as a function of background intensity for Series B and C. The data can be approximated by a square-root relationship. For Series $B$ the $\sigma_{1}$ values are related to the average slope of the psychometric curves or to the response probabilities at the three higher flash levels only. The latter $\sigma_{I}$ values tend to be lower.

background. As this variation is nonmonotonous, a particular differential sensitivity can be caused by two different background intensities. Detection of a flash presented on a background yields a square-root relation between the differential sensitivity measure and the background intensity.

\section{DISCUSSION}

In this paper we have again used a measure of sensitivity, $\sigma_{I}$ (cf. Eijkman et al, 1966), that is different from the commonly used increment threshold $\Delta \mathrm{I}$. The value of $\sigma_{\mathrm{I}}$ is determined by the slope of the psychometric curve. Thijssen and Vendrik (1968) showed that in general this slope changes by shifting the curve vertically upwards or downwards. In terms of the theory of signal detection, a shift of the psychometric curve is caused by a shift of the criterion level. However, the change of the slope appeared to be a second-order effect in comparison with the horizontal shift resulting from a criterion shift (Thijssen, 1969). As may be illustrated by the results of Blackwell (1963), the values of $\sigma_{\mathrm{I}}$ and $\Delta_{\mathrm{I}}$ are proportional, at least on an average tor several Os. This property may be expressed by the Crozier ratio (Crozier, 1936), $\Delta \mathrm{I} / \sigma_{\mathrm{I}}$, which equals about 3 for the data of Blackwell. However, from the data of Mueller (1951), it can be deduced that $\Delta \mathrm{I} / \sigma_{\mathrm{I}}=6$. This result agrees with the statement above, that a criterion maintained at a constant level yields a proportionality of $\sigma_{I}$ with $\Delta \mathrm{I}$, but every criterion level corresponds with a particular $\Delta \mathrm{I}$ value. Therefore, $\sigma_{\mathrm{I}}$ will be a more convenient measure of sensitivity than the increment threshold $\Delta \mathrm{I}$.

The proportionality of $\sigma_{I}$ to flash intensity (Fig. 6) which is obtained for Series B displays a constant Crozier ratio, because flash intensity stands for increment threshold $\Delta I$ [i.e., $\left.P\left(R_{2} \mid I_{j}\right)=0.50\right]$. Comparison with the results of the three series shows, however, that the proportionality also holds in case of no identity of flash intensity and increment threshold. This result was also obtained by Cornsweet and Pinsker (1965), and by Leshowitz et al (1968) with a somewhat different stimulus configuration. This very strongly suggests that it is reasonable to call the proportionality of $\sigma_{1}$ to flash intensity by its proper name, i.e., Weber's law. In vision, the name of Weber is mostly connected with the proportionality of the increment threshold to the background intensity, and this proportionality holds at photopic intensity levels. A lot of models were proposed to account for this property (cf. Bouman et al, 1963; Barlow, 1965; Treisman, 1966; Van der Grind et al, 1970; Koenderink et al, 1970), but much less for the proportionality of the differential sensitivity to the flash intensity.

Cornsweet and Pinsker (1965) showed that a logarithmic transducer might account for Weber's law, assuming that with dark-adapted retina (cf. Series A) the noise level is constant. However, such a transducer is not very likely (Thijssen, 1969; Treisman, 1966). Moreover, Weber's law holds also under conditions of light adaptation (Series D) and the presence of a b a c k g r o und (S e ries C). Electrophysiological measurements indicate that fluctuations in neural activity at maintained stimulation increase slightly with increasing light intensity at low levels, become constant at intermediate levels, and even decrease at high levels (cf. Barlow \& Levick, 1969; and also by combining the results of Dodge et al, 1968, and of Hagins, 1965).
Treisman and Leshowitz (1969) extended the model of Treisman (1966) to pedestal procedures which are equivalent to the present procedure. The essence of the model is that a single ganglion cell delivers a number of sensory messages, each consisting of a number of spikes, which are positively correlated. The total resulting fluctuations are composed of two independent noise sources, i.e., the quantum fluctuations of light, and the variability of the individual sensory messages. In our opinion, a ganglion cell will yield only one sensory message when the stimulus characteristics are within the spatial and temporal summation limits. Hence, Treisman's model is not very useful in explaining the results of our experiments. Moreover, the linear transducer implied in the model (Treisman \& Leshowitz, 1969) is not likely to be applicable in our experiments (Thijssen, 1969).

A third model, i.e., the multirange meter model, to account for Weber's law has been mentioned earlier. This model was first applied to auditory discrimination experiments (Eijkman et al, 1966) and extended later to visual discrimination (Thijssen \& Vendrik, 1968; Thijssen, 1969). The model is shown in Fig. 9 and consists of a transducer with a variable gain, a noise source that adds its output to the output of the transducer, and a detector that operates according to the description given in Psychophysical Methods above. With this model, Weber's law can be explained by assuming that the gain of the transducer is adapted to the range of stimulus intensities in order to maintain the input of the detection mechanism within its dynamic range. It is assumed that this range setting is performed during the introductory period 


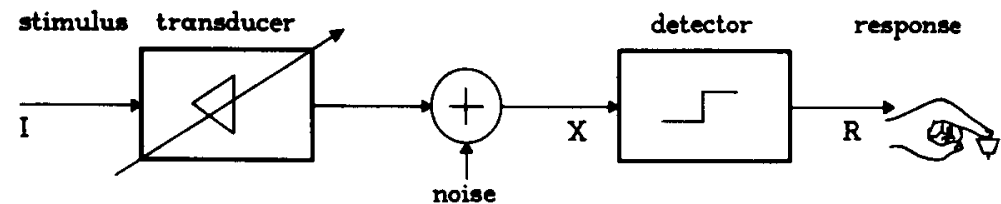

Fig. 9. The multirange meter model. The gain of the transducer is adapted according to the intensity level of the stimuli. The decision variable, $X$, is maintained within a fixed range by this mechanism. Additive noise is present.

which precedes an experiment. When the mean output of the transducer is always set at a fixed level, it can be shown that, with a constant noise level, Weber's law will be measured at the input of the transducer, i.e., in stimulus magnitude (see Appendix).

It will be clear that it makes no essential difference in this model whether a range-setting mechanism is activated by a training procedure or by a background intensity. Hence, these two mechanisms may operate simultaneously.

The influence of a background can be investigated with Figs. 6 and 7. Figure 6 permits the conclusion that the differential sensitivity is largest (i.e., $\sigma_{\mathrm{I}}$ is small) for Series $\mathrm{C}$. This is compatible with the data shown in Fig. 7. In the latter figure it appears that, unless the masking effect of the background becomes important, the value of $\sigma_{\mathrm{I}}$ becomes smaller with increasing background intensity. If background intensity and adaptation level are assumed to be identical in their effect on the sensitivity (cf. Crawford, 1947; Blakemore \& Rushton, 1965), the displacement of the $\sigma_{\mathrm{I}}$ vs flash intensity line in Fig. 6 for Series D with respect to Series C is also in agreement with the results shown in Fig. 7 .

The parallel displacement of the lines in Fig. 6 with changes of background intensity and adaptation level suggests an adaptation mechanism acting like a variable gain factor. Such a gain-control mechanism is very attractive because the relative difference between the various stimuli remains constant. It will be shown below that an additional property is necessary to explain the results completely. The idea of a gain control in the visual system has been proposed by Craik (1940), who compared light adaptation with the range-setting switch of a multirange meter. Psychophysical data to support the concept can be obtained from papers of Craik (1940) and of Stevens and Stevens (1963). These authors showed, in binocular matching experiments, that the flash response curve adapts proportionally to the square root of the background intensity.

Psychophysical results were confirmed recently in electrophysiological experiments carried out by Sakman and Creutzfeld (1969) and Glantz (1968). Sakman and Creutzfeld measured the flash-response curves of ganglion cells in the retina of the cat and Glantz measured graded potentials in the photoreceptor of the crayfish. Both papers clearly demonstrate a parallel shift with background intensity of the flash-response curves in a log-log plot; moreover, the shift is proportional to the square root of the background intensity. Hence, in order to maintain a fixed response level, the flash intensity has to be changed with a factor proportional to the square root of the background intensity.

The results of the papers quoted above may explain the data of Series B (Fig. 8), i.e., the square-root relation, but only partially the results of the other series. However, a further inspection of the flash response curves from electrophysiological data (see also Büttner \& Grüsser, 1968; Stone \& Fabian, 1968; Fatehchand et al, 1962) shows that these curves display a narrow dynamical range. A saturation effect starts 1 to $2 \log$ units after the first observable response. This proporty of the retina cells may explain why the $\sigma_{\text {I }}$ values of Series A (dark-adapted retina) differ only slightly from those of Series C and D and even are somewhat larger: the flash response mechanism operates in the saturation range with a dark-adapted retina. Now the data of Fig. 7 become understandable. The increase of $\sigma_{\mathrm{I}}$ which should be observed with increasing background intensity is reversed by the fact that the flash response mechanism is forced out of the saturation range. Assuming that the increment flashes in Series B evoke a response that is proportional to the flash intensity, i.e., $\Delta \mathrm{X}($ :) $\Delta \mathrm{I}$, but also inversely proportional to the square root of background intensity, i.e., $\Delta \mathrm{X}(:)(I)^{-1 / 2}$, the observed square-root relation has been explained if the noise level is independent of the background intensity (cf. Barlow and Levick, 1969).

An alternative explanation of the results shown in Figs. 6-8 by the quantum fluctuation concept is not very attractive. This concept does not agree with the proportionality of $\sigma_{\mathrm{I}}$ to flash intensity or with the decrease of $\sigma_{I}$ values at application of a background (Series $\mathrm{C}$ as compared with Series A and D; see also Fig. 7). Leshowitz et al (1968) and
Cornsweet and Pinsker (1965) also derived data which are not in agreement with the quantum fluctuation concept. The electrophysiological data of Barlow and Levick (1969) for ganglion cells of the cat show that fluctuations in response to a steadily presented field are constant over a large intensity range and even decrease at higher levels. They conclude that the increment threshold curve (i.e., the square-root relation) is most probably caused by adaptation of the flash response.

It appears that the multirange meter concept that was originally proposed by Eijkman et al (1966) to explain the results of auditory experiments is very useful in describing the influence of background intensity or of adaptation level on the differential sensitivity for the discrimination of two flashes. It has to be assumed that the sensitivity switch is operated in a particular way by the background intensity; this assumption is confirmed by psychophysical and electrophysiological data. The proportionality of $\sigma_{I}$ to flash intensity, which is called Weber's law, has been explained by the multirange meter model also, but some crucial experiments are being performed now. The results of these experiments will be presented in a subsequent paper, together with a more sophisticated version of the model.

\section{APPENDIX}

The multirange meter model (see also Fig. 9) can be formalized by the equation

$$
\mathrm{X}=\alpha \mathrm{F}(\mathrm{I})+\beta
$$

with $\mathrm{X}$ being the (fluctuating) decision variable, $\alpha$ being the gain factor of the transducer, $F(I)$ being called the transducer function, which relates the mean value of $X$ to stimulus intensity, $\beta$ representing the noise, which causes $X$ to fluctuate, and being normally distributed.

Consequently,

$$
\begin{gathered}
\langle\mathrm{X}\rangle=\alpha \mathrm{F}(\mathrm{I})+\langle\beta\rangle \\
\sigma_{\mathrm{X}}=\sigma_{\beta}
\end{gathered}
$$

The range-setting mechanism, which manipulates the gain $\alpha$, has to maintain $\mathrm{X}$ in a fixed range. Therefore, when assuming $\langle\beta\rangle=$ constant it follows:

$$
\alpha \mathrm{F}(\mathrm{I})=\text { constant }
$$

Two I values, corresponding to the response probabilities 0.50 and 0.84 , respectively, can be determined experimentally. Call them $\mathrm{I}$ and $\mathrm{I}+\sigma_{\mathrm{I}}$. As these stimuli are used in the same experiment, $\alpha$ will be identical at the presentation of each of the stimuli, so: 


$$
\begin{aligned}
\langle\mathrm{X}\rangle & =\alpha \mathrm{F}(\mathrm{I})+\langle\beta\rangle \\
\langle\mathrm{X}+\Delta \mathrm{X}\rangle & =\alpha \mathrm{F}\left(\mathrm{I}+\sigma_{\mathrm{I}}\right)+\langle\beta\rangle
\end{aligned}
$$

Now $\langle X+\Delta X\rangle-\langle X\rangle=$ constant because in every experiment the region corresponding to $\mathrm{P}(0.50,0.84)$ is taken, and therefore $\Delta \mathrm{X}$ equals $\sigma_{\mathrm{X}}$, which is constant in this model. With Eq. 4 the difference can be written:

$$
\alpha\left[F\left(I+\sigma_{I}\right)-F(I)\right]=\sigma_{X}=\text { constant }
$$

and with Eq. 3:

$$
\left[F\left(I+\sigma_{I}\right)-F(I)\right] / F(I)=\text { constant }
$$

or:

$$
\left[\mathrm{F}\left(\mathrm{I}+\sigma_{\mathrm{I}}\right)\right] / \mathrm{F}(\mathrm{I})=\mathrm{constan} t
$$

When a power function [i.e., $F(I)=I n$ ] is inserted in Eq. 5 (cf. Treisman, 1966) it follows:

$$
\left(\mathrm{I}+\sigma_{\mathrm{I}}\right)^{\mathrm{n}} / \mathrm{I}^{\mathrm{n}}=\text { constant }
$$

or:

$$
\left(1+\sigma_{\mathbf{I}} / \mathrm{I}\right)^{\mathrm{n}}=\text { constant } .
$$

Hence, $\sigma_{\mathrm{I}} / \mathrm{I}$ has to be constant, so Weber's law is valid. This result is independent of the value of $n$.

\section{REFERENCES}

BARLOW, H. B. Increment thresholds at low intensities considered as signal noise discriminations. Journal of Physiology, 1957, $136,469-488$

BARLOW, H. B. Optic nerve impulses and Weber's law. In: Cold Spring Harbor symposia on quantitative biology. Cold Spring Harbor, L.I., N.Y., 1965, 30, 539-546.

BARLOW, H. B., \& LEVICK, W. R. Three factors limiting reliable detection of light by retinal ganglion cells of the cat. Journal of Physiology, 1969, 200, 1-24.

BLACKWELL, H. R. Neural theories of simple visual discrimination. Journal of the Optical Society of America, 1963, 53, 129-160.

BLAKEMORE, C. B., \& RUSHTON, W. A. H. The rod increment threshold during dark adaptation in normal and rod monochromat. Journal of Physiology, 1965, 181, 629-640.

BOUMAN, M. A. Peripheral contrast thresholds for various and different wavelengths for adapting field and test stimulus. Journal of the
Optical Society of America, 1952, 42, 820-831.

BOUMAN, M. A., \& van den BRINK, G. On the integrative capacity in time and space of the human peripheral retina. Journal of the Optical Society of America, 1952, 42, 617-620.

BOUMAN, M. A., VOS, J. J., \& WALRAVEN, P. L. Fluctuation theory of luminance and chromaticity discrimination. Journal of the Optical Society of America, 1963, 53, 121-128.

BÜTTNER, U., \& GRÜSSER, O. J. Quantitative Untersuchungen der rä̈mlichen Erregungssummation im rezeptiven Feld retinaler Neurone der Katze. I. Kybernetik, $1968,4,81-94$.

CORNSWEET, T. N., \& PINSKER, H. M. Luminance discrimination of brief flashes under various conditions of adaptation. Journal of Physiology, 1965, 176, 294-310.

CRAIK, K. J. W. The effect of adaptation on subjective brightness. Proceedings of the Royal Society, B, 1940, 128, 232-247.

CRAWFORD, B. H. Visual adaptation in relation to brief conditioning stimuli. Proceedings of the Royal Society, B, 1947, 134, 283-302.

CROZIER, W. J. On the variability of critical illumination for flicker fusion and intensity discrimination. Journal of General Physiology, 1936, 19, 503-522.

DENTON, E. J., \& PIRENNE, M. H. Spatial summation at the absolute threshold of peripheral vision. Journal of Physiology, 1952, 116, 32P-33P.

DODGE, F. A., KNIGHT, B. W., \& TOYODA, J. Voltage noise in Limulus visual cells. Science, $1968,160,88-90$.

EIJKMAN, E., THIJSSEN, 3. M., \& VENDRIK, A. J. H. Weber's law, power law, and internal noise. Journal of the Acoustical Society of America, 1966, 40, 1164-1173.

EIJKMAN, E., \& VENDRIK, A. J. H. Detection theory applied to the absolute sensitivity of sensory systems. Biophysiological Journal, 1963, 3, 65-78.

FATEHCHAND, R., LAUFER, M., \& SVAETICHIN, G. Retinal receptor potentials and their linear relationship to light intensity. Science, 1962, 137, 666-667.

GLANTZ, R. M. Light adaptation in the photoreceptor of the crayfish. Vision Research, 1968, 8, 1407-1421.

GREEN, D. M., \& SWETS, J. S. Signal detection theory and psychophysics. New York: Wiley, 1966.

HAGINS, W. A. Electrical signs of information flow in photoreceptors. In: Cold Spring Harbor symposia on quantitative biology, 1965. Cold Spring Harbor, L.I., N.Y., 1965, $30,403-418$.

KOENDERINK, J. J., VAN de GRIND, W. A., \& BOUMAN, M. A. Models of retinal signal processing at high luminances. Kybernetik, 1970, 6, 227-237.
LESHOWITZ, B., TAUB, H. B., \& RAAB, D. H. Visual signals in the presence of continuous and pulsed background. Perception \& Psychophysics, 1968, 4, 207-213.

MUELLER, C. G. Frequency of seeing functions for intensity discrimination at various levels of adapting intensity. Journal of General Physiology, 1951, 34, 463-474.

PIRENNE, M. $H$. The visual process. In $\mathrm{H}$. Davson (Ed.), The eye. Vol. 2. New York and London: Academic Press, 1962.

SAKM AN, B., \& CREUTZFELD, O. D. Scotopic and mesopic light adaptation in the cat's retina. Pflügers Archiv, 1969, 313, 168-185.

STEVENS, J. C., \& STEVENS, S. S. Brightness function: Effects of adaptation. Journal of the Optical Society of America, 1963, 53, 375-385.

STONE, J., \& FABIAN, M. Summing properties of the cat's retinal ganglion cells. Vision Research, 1968, 8, 1023-1040.

SWETS, J. A. Signal detection and recognition by human observers: Contemporary readings. New York: Wiley, 1964.

THIJSSEN, J. M. Differential luminance sensitivity of the human visual system. A detection theoretical model based on psychophysical and electrophysiological data. Thesis Nijmegen University, 1969. (Nijmegen: Drukkerij Gebr. Janssen N.V.)

THIJSSEN, J. M., \& VENDRIK, A. J. H. Internal noise and transducer function in sensory detection experiments: Evaluation of psychometric curves and of ROC curves. Perception \& Psychophysics, 1968, 3, $387-400$.

TREISMAN, M. A statistical decision model for sensory discrimination which predicts Weber's law and other sensory laws: Some results of a computer simulation. Perception \& Psychophysics, 1966, 1, 203-230.

TREISMAN, M., \& IRWIN, R. J. Auditory intensity discriminal scale. I. Evidence derived from binaural intensity summation. Journal of the Acoustical Society of America, 1967, 42, 586-592.

TREISMAN, M., \& LESHOWITZ, B. The effects of duration, area, and background intensity on the visual intensity difference threshold given by the forced choice procedure: Derivations from a statistical decision model for sensory discrimination. Perception \& Psychophysics, $1969,6,281-296$.

VAN de GRIND, KOENDERINK, I. J., \& BOUMAN, M. A. Models of the processing of quantum signals by the human peripheral retina. Kybernetik, 1970, 6, 213-227.

ZIGLER, M. J., \& WOLF, E. Uniocular and binocular scotopic parafoveal sensitivity. American Journal of Psychology, 1958, 71, 186-198.

(Accepted for publication November 1, 1970.) 